\title{
Preventing Bladder Recurrences after Nephroureterectomy for Upper Track Urothelial Cancer
}

\author{
Edward M. Messing* \\ University of Rochester Medical Center, Rochester, NY, USA
}

Received/Accepted 4 April 2019

Bladder recurrences of urothelial cancer after nephroureterectomy and bladder cuff excision (NU) for upper tract (UC) occur in about $30 \%$ of patients who've not had prior bladder cancers, with most of these occurring in the first year after surgery [14]. A single intravesical instillation of chemotherapy immediately after a transurethral resection of bladder tumor (TURBT) can significantly reduce the likelihood of tumor recurrence using a variety of agents (summarized in ref \# 5) [5], but does it help after NU for UT UC? And if so, what agent should be used and when should it be administered?

The most compelling article providing level 1 evidence supporting this practice was a randomized prospective study carried out in the United Kingdom and published in 2011 [1]. In this study, with followup of 12 months in the intent-to-treat analysis, 21 of $120(17 \%)$ patients without prior bladder tumors randomized to mitomycin (MMC) and 32 of 119 $(27 \%)$ patients in the standard care arm experienced bladder cancer recurrences by 12 months $(p=0.055)$. Secondary analysis of patients treated per protocol revealed $16 \%$ in the MMC group versus $27 \%$ in the standard group $(p=0.03)$ had bladder recurrences.

\footnotetext{
${ }^{*}$ Correspondence to: Edward M. Messing, MD, FACS, University of Rochester Medical Center, Rochester, NY, USA. Tel.: +1 585275 3345. E-mail: Edward_Messing@urmc.rochester.edu.
}

The strengths of the study include it's randomized prospective design, and that no patient had a history of prior or concomitant bladder cancer, so the influence of treatment was not "contaminated" by the effects of prior bladder tumors or tumor recurrences. Also, the instillation was performed at the time of catheter removal, usually 10-14 days post operatively, to avoid concerns about immediate postoperative extravasation of the instilled drug. There were no serious adverse events attributed to the drug in those who received treatment.

A major weakness however, was that the diagnosis of recurrence did not require histological confirmation (cystoscopic appearance alone was sufficient). A second weakness was that owing to its design (saline or water instillation was not used in the control arm) neither patients nor, more importantly, urologic surgeons were blinded to treatment. This may have influenced the reporting of recurrences, particularly when histologic confirmation of recurrence was not required (and how many patients had biopsy proven recurrence in either arm was not reported). Another problem was that nearly $16 \%$ of the 284 patients originally randomized and almost $23 \%$ in the per protocol analysis (the analysis with a statistically significant reduction in bladder cancer recurrences) were not included in the intent-to-treat analysis. While the explanations for these exclusions seemed reasonable 
(e.g. from not having UT UC at surgery - since a biopsy before NU wasn't required, to dying postoperatively or during the follow-up), and reflect real life issues, it still detracts from both analyses. Furthermore, if one of the benefits of immediate post TURBT intravesical instillation therapy had been hypothesized to be killing floating tumor cells and preventing their implantation, $[4,5]$ whether this was still occurring two weeks after NU is uncertain.

In a separate multi-institutional randomized phase 2 study from Japan, $30 \mathrm{mg}$ Pirarubicin (THP) in $30 \mathrm{ml}$ saline held for 30 minutes, was instilled within 48 hours after NU (vs no instillation) in patients with no prior or concomitant history of bladder cancer. Ito, et al reported that $16.9 \%$ of 36 patients receiving THP had bladder recurrences at 12 and 24 months of follow-up, while $31.8 \%$ and $42.2 \%$ of 36 patients receiving no instillation had recurrence by 12 months and 24 months, respectively $(p=0.025)$ [6]. In a multivariate analysis of demographic, tumor and surgical characteristics, only receiving THP or having open (as opposed to laparoscopic) surgery, predicted significantly fewer bladder cancer recurrences. THP instillations were well tolerated.

This study had less than one-third the number of subjects in O'Brien et al's study [1] and also was not a double blind, placebo controlled trial. However, it required histological confirmation of bladder cancer recurrences, reducing some of the potential biases in O'Brien et al's trial [1].

These are only the only randomized prospective trials in this area. Most other studies are decades long, non-randomized single institution reports $[3,7]$ and in the largest, treatment consisted of courses of 6 or more intravesical instillations of chemotherapy starting two weeks after surgery. MMC, epirubicin and THP have been used in these studies without differences in efficacy for one agent or the other.

If the rationale for withholding postoperative instillation for 10-14 days (chosen to avoid complications) could be questioned in terms of mode of action, both Noennig [3] and Moriarty [2] and their respective coworkers have utilized intraoperative administration with complete safety (although only one of these studies reported efficacy) [3]. The medications, usually MMC (but Moriarty, et al [2] also used Doxirubicin) were instilled in the same concentrations and volumes utilized for standard post TURBT intravesical instillations, during NU (minimally invasive or open) for 45-60 minutes. Instilled drug was drained out and the bladder irrigated with $100 \mathrm{~mL}$ saline before the bladder cuff was taken. In Noennig, et al's [3] non-randomized series, patients receiving an intraoperative MMC instillation had (non-significantly) fewer recurrences at a year than those who received it on post-operative days $1-3(p=0.09)$, although the benefits seemed more in delaying time to recurrence than in preventing overall recurrences [3]. Instillations at both timepoints (intraoperative vs postoperative day 1-3) were tolerated well.

Besides its non-randomized design, making the results of this series even more difficult to interpret was that over $33 \%$ of patients had histories of prior bladder tumors, and over half of those had previously received intravesical therapies. The specifics of these details and their impacts on bladder tumor recurrence were not reported. An additional question is that if one clips or blocks the ureter below the suspected tumor, [2] why would an intraoperative chemotherapy instillation be more beneficial than one given 1-3 days later? It is possible that in the case of multifocal cancer, or carcinoma-in-situ, particularly below the site of ureteral occlusion, tumor cells could move downstream with ureteral manipulation before tumor removal, but location of UTUCs and their impact on bladder tumor recurrences was not reported by Noennig, et al. [3].

In summary, while the majority of studies show a benefit in preventing UC recurrences in the bladder for an immediate (or soon after surgery) intravesical instillation of chemotherapy after (or during) NU for UT UC, only half of respondents to a survey of members of the Society of Urologic Oncology said that they use this treatment [8]. All published studies report safety of the instillation, but except for the randomized studies, in how many patients instillation was withheld is uncertain. Many questions, including best agents, when they should be instilled, and the magnitude of effect are uncertain/variably reported. The rarity of this condition makes meaningful single institution studies of limited value and begs for well-designed, randomized, prospective multiinstitutional/cooperative group trials to address these questions.

\section{ACKNOWLEDGMENTS}

The authors have no acknowledgments.

\section{FUNDING}

The authors report no funding. 


\section{CONFLICTS OF INTEREST}

Nothing to disclose.

\section{REFERENCES}

[1] O'Brien T, Ray E, Singh R, et al. Prevention of bladder tumors after nephroureterectomy for primary upper urinary tract urothelial carcinoma: A prospective, multicenter randomized clinical trial of a single post-operative intravesical dose of Mitomycin C (the OD MUT-C trial). Eur Urol. 2011;60:703-710.

[2] Moriarty MA, Uhlman, MA, Bing MT, et al. Evaluating the safety of intraoperative instillation of intravesical chemotherapy at the time of nephroureterectomy. BMC Urology. 2015;15:45.

[3] Noennig B, Bozorgmehri S, Terry R, et al. Evaluation of intraoperative versus postoperative adjuvant Mitomycin C with nephroureterectomy for urothelial carcinoma of the upper urinary tract. Bladder Cancer. 2018;4:389-394.

[4] Ito A, Shintaku I, Satoh M, et al. Intravesical seeding of upper urinary tract urothelial carcinoma cells during nephroureterectomy: An exploratory analysis from the THPMG trial. Jpn J Clin Oncol. 2013;43:1139-1144.

[5] Messing EM, Tangen CM, Lerner SP, et al. Effect of Intravesical Instillation of Gemcitabine vs Saline Immediately Following Resection of Suspected Low-Grade Non-Muscle-Invasive Bladder Cancer on Tumor Recurrence SWOG S0337 Randomized Clinical Trial. JAMA. 2018;319 (18):1880-1888. doi:10.1001/jama.2018.4657

[6] Ito A, Shintaku I, Satoh M, et al. Prospective randomized phase II trial of a single early intravesical instillation of Pararubicin (THP) in the prevention of bladder recurrence after nephroureterectomy for upper urinary tract urothelial carcinoma: The THP Monotherapy Study Group Trial. J Clin Onc. 2013;31:1422-1427.

[7] Wu W-J, Ke H-L, Yang Y-H, et al. Should patients with primary upper urinary tract cancer receive prophylactic intravesical chemotherapy after nephroureterectomy? J Urol. 2010;183:56-61.

[8] Lu DD, Boorjian SA, Raman JD. Intravesical chemotherapy use after radical nephroureterectomy: A national survey of urologic oncologists. Urol Onc. 2017;35:113. 